\title{
Organízate con eficacia para jóvenes.
}

\section{Get organized effectively for young people.}

Toma el control de tu vida en un mundo de distracciones.

Take control of your life in a world of distractions.

Adriana L.M. Montiel-Ávila ${ }^{a}$,Carlos J. Benítez-Víquez ${ }^{b}$

\begin{abstract}
:
This book aims to help young people improve their competence in the basic skills they need to fase life effectively. These skills will help them not to suffer so many distractions and experience less stress, at the same time that they will see their confidence in themselves and in themselves increase, their presence, productivity, creativity and fun. Through the Method Organize effectively (GTD). The book is organized in three parts: The art of organizing effectively, organize effectively in practice and in the laboratory.
\end{abstract}

\section{Keywords:}

Method, youth and efficiency

\section{Resumen:}

El presente libro tiene como objetivo ayudar a los jóvenes a mejorar su competencia en las habilidades básicas que necesitan para afrontar la vida con eficacia. Esas habilidades les ayudarán a no sufrir tantas distracciones y a experimentar menos agobio y estrés, al mismo tiempo que verán incrementarse su confianza en sí mismos y en sí mismas, su presencia, productividad, creatividad y su diversión. A través del Método Organízate con eficacia (GTD) El libro está organizado en tres partes: El arte de organizarse con eficacia, organízate con eficacia en la práctica y el laboratorio.

Palabras Clave:

Método, jóvenes y eficacia.

\section{Desarrollo}

Para iniciar la presente obra los autores cuestionan ¿Qué significa estar listo? Para dar respuesta es necesario definir la expresión como una combinación de cuatro aspectos:

1. Listo para el ahora. Lo que implica mostrar una buena disposición a prestar atención, ahora mismo, en el momento presente.
2. Listo para lo que viene a continuación. Requiere ser plenamente consciente de las cosas qué debe hacer durante un determinado periodo de tiempo.

3. Listo para las transiciones. Conlleva tener la capacidad de gestionar los cambios en áreas clave de la vida. Y

4. Listo para cualquier cosa. Comprende afrontar la vida con tranquilidad y sentirse a punto, para lo que sea que salga al paso.

a Universidad Autónoma del Estado de Hidalgo, programa educativo de administración. Instituto de Ciencias Económico Administrativas, http://orcid.org/0000-0003-2286-2843,CCorreo electrónico adriana_montiel1194@uaeh.edu.mx

b Universidad Autónoma del Estado de Hidalgo, programa educativo de administración, Instituto de Ciencias Económico Administrativas, http://orcid.org/0000-0001-8668-1991 Correo electrónico carlos.viquez@ gmail.com 
Asimismo, resaltan que el cerebro es la herramienta más poderosa y compleja que poseen los jóvenes, para ello hay que identificar las dos áreas del cerebro.

La amígdala, que es responsable de las reacciones instantáneas y la otra, denominada córtex prefrontal que se encarga del pensamiento más reflexivo y de la toma de decisiones, por lo tanto, es responsable de resolver los problemas y de sintetizar, así como de dar sentido a todo lo que se ve y se hace.

\section{Primera parte. El arte de organizarse con eficacia.}

El Método Organízate con eficacia es un método que consiste en aprender a centrarse y a implicarse en el presente, siendo consciente de lo que viene a continuación, busca la estabilidad cuando las cosas se descontrolan e imagina con ilusión lo que la vida puede ofrecer en cualquier aspecto: estudios, trabajo, relaciones, deportes, objetivos, sueños, entre otros.

Para comprender como funciona el GTD los autores hacen alusión a los siguientes temas:

1. El Problema. Se refiere a la complejidad de los cambios que suceden en la vida algunos llegan en el momento oportuno, otros antes de que se esté preparado, y se cuestiona:

¿Qué siento ahora mismo en relación con las complicaciones de mi vida?

¿Sientes que te desbordan?

¿Te estresan?

¿O necesitas un nuevo desafío?

¿Tal vez estas aburrido o aburrida a más no poder?

¿O quizás algunos ámbitos de tu vida te sobrepasan, mientras que otros te arrancan bostezos?

2. Conectividad y flujo de información. Al respecto los autores expresan que en toda la historia, los seres humanos han estado tan conectados como en la actualidad. Esa conectividad constante ha transformado la manera de estudiar, trabajar, comprar, viajar, relacionarse y comunicarse. También ha transformado los pensamientos y la manera de pensar. Actualmente los jóvenes tienen integrada la conectividad digital masiva.

Y cuestionan: ¿Qué vas a necesitar para salir airoso? para ello sugieren que la respuesta está en la capacidad de pensar, de concebir nuevas ideas y ponerlas en práctica. El pensamiento es la destreza que siempre será necesaria. Ayudará en el momento presente y definirá lo que se hará al siguiente momento. Dentro de pocos años, le servirá para encontrar trabajo.

Para lograrlo los autores reconocen dos trampas: Sobrecarga y agobio, y la distracción. Este libro ofrecerá herramientas para evitar dichas trampas.

1. La promesa "Estado ready". Es la capacidad de pensar y controlar la atención. Cuando se domina la atención, eres capaz de relajarte y dedicar la cantidad adecuada de energía y concentración a lo que estés haciendo. Es entonces cuando entras en un estado ready.

Ejemplos de estados ready: Dispuesto a aprender, Lista para hacer los deberes, Listo para el colegio, A punto para relajarse, Dispuesto a trabajar, Preparado a escuchar, Listo para actuar, entre otros,

2. En la práctica. Cuando sabes que puedes alcanzar un estado ready sean cuales sean las circunstancias, puedes afrontar cualquier situación sin miedo ni preocupación, listo o lista para responder y generar acontecimientos. ¿Verdad que sería genial estar ready siempre que hiciera falta?

3. Asuntos. Es algo que aparece en tu vida física, digital, mental o emocional y que requiere algún tipo de decisión o acción por tu parte que no está previamente decidida ni organizada. Ejemplos: Exámenes, divorcio de los padres, problemas de salud, amigos, redes sociales, presión de los padres, salir con alguien, entre otros.

4. Control y perspectiva. El control ayudará a crear un espacio mental para pensar e imaginar y a despejar tu mundo con el fin de poderte concentrar en los temas que más te motivan. El control solamente es la primera parte de la fórmula que propicia el estado ready. También debes saber a dónde vas y por qué, A esto se le llama perspectiva que es la capacidad de mirar adelante para ver a dónde te diriges. Es la visión, el porqué de todo aquello que decides hacer.

5. El hábito productivo. Para que tomes el control de tus asuntos, los autores sugieren aprender cinco pasos:

- Capturar,

- Clarificar,

- Organizar,

- Reflexionar y

- Ejecutar. 


\section{Segunda parte: Organízate con eficacia en la práctica.}

El método Organízate con eficacia consta de tres áreas principales:

La primera área comprende cinco pasos para ayudar a tomar el control: Capturar lo que atrapa tu atención, clarificar que significa cada asunto para ti y decidir qué hacer al respecto, organizar los resultados en categorías, reflexionar sobre el contenido de nuestro sistema de poder, y ejecutar y poner manos a la obra con confianza siempre que sea necesario. A continuación, se conceptualiza cada uno de ellos.

1. La captura tiene como herramientas: bandeja de entrada, herramientas de captura, lista detonante y primer inventario. Cada una con actividades específicas referentes a los espacios físico, digital y mental.

2. Clarificar es el paso más importante de los cinco, el más difícil y profundo, tiene como herramienta la denominada de transformación con seis categorías: Acciones, proyectos, listas de verificación, algún día/tal vez, material de referencias y basura

3. Organizar contempla sus herramientas como son los Mapas (Calendario, lista de acción siguiente, Lista de Proyectos) Lista de comprobación, Lista de algún día/tal vez y archivo de referencia.

4. Reflexionar presenta como herramientas la revisión diaria, revisiones semanales y los niveles de enfoque

5. Ejecutar se apoya en la estrategia denominada el foco con cuatro criterios:

- Ubicación,

- tiempo,

- energía y

- $\quad$ prioridad

Segunda área llamada: Cómo adquirir perspectiva: niveles de enfoque.

Los niveles de enfoque ayudarán a capturar, clarificar y gestionar la atención y perspectiva a largo del tiempo. Los niveles de enfoque son:

1. El propósito

2. Visión

\section{Objetivos}

4. Áreas de enfoque:

- Bienestar

- Familia

- Estudios

- Actividades deportivas

- Primeros trabajos, entre otros

5. Proyectos y

6. Acciones

Tercera sección corresponde al Proyecto planificación (El mapa de planificación) Es una herramienta de pensamiento sencilla pero potente, en virtud que planifica procesos de una manera rápida y fluida, aporta claridad a cualquier proyecto, situación e intención y consta de cinco pasos:

1. Definir el propósito, los principios y las condiciones

2. Visualizar el resultado

3. Lluvia de ideas

4. Organizar

5. Identificar las accione

Tercera parte el Laboratorio. Los autores proporcionan una serie de ejemplos para dar mayor claridad a lo expuesto anteriormente, relacionados con las áreas de enfoque y que son de interés para los jóvenes. Asimismo, incluyen con un glosario d ellos términos que se utilizan en la presente obra, y para finalizar incluyen un índice visual, que retroalimenta los contenidos del libro y sobre todo el Método Organízate con eficacia

\section{Bibliografía}

[1] ALLEN, D., WILliaMS, M. \& WALlaCE, M. (2018) Organízate con eficacia (Getting Things Done) para jóvenes. Toma el control de tu vida en un mundo de distracciones. $1^{\text {a }}$ edición. Empresa Activa. Barcelona, España. 